\title{
Evaluating the Effect of Asymmetric Cross-section in Free Vibration and Bending Analysis Results of FG Sandwich Beam by Proposing Simple Efficient Element
}

\author{
Majid Yaghoobi ${ }^{1 *}$, Mohsen Sedaghatjo ${ }^{1}$, Reyhaneh Alizadeh${ }^{1}$, Mohammad Karkon $^{2}$ \\ ${ }^{1}$ Civil Engineering and Architecture Department, Engineering Faculty, University of Torbat Heydarieh, Torbat Heydarieh \\ 95161-68595, Iran \\ 2 Civil Engineering Department, Larestan Branch Islamic Azad University, Larestan 74318-95659, Iran \\ *Corresponding author, e-mail: majidyaghoobi@torbath.ac.ir
}

Received: 04 November 2020, Accepted: 01 February 2021, Published online: 19 February 2021

\begin{abstract}
In this paper, the asymmetric effect of the cross-section on the free vibration and bending analysis of FG sandwich beams are evaluated. For this purpose, a simple, efficient element is formulated. The new element is created based on the Timoshenko beam theory. The third- and second-order polynomials will be used for vertical displacement and rotation fields, respectively. The proposed formulation will be written based on satisfying the equilibrium equation. Satisfying the equilibrium equation of the Timoshenko beam, in addition to increasing element efficiency, will reduce the number of nodal unknowns. Several benchmark tests with different boundary conditions are used for thin and thick beams to prove the efficiency of the proposed element. The responses of the good elements of other researchers have been used for comparison. Numerical tests prove the rapid convergence rate and high accuracy of the proposed element in free vibration and bending analysis of the beams with various cross-section types and different boundary conditions. The pinned-sliding support conditions for the beam are used to evaluate the asymmetric effect of the cross-section. The use of asymmetric cross-sections creates additional axial displacements and intensifies the deflection of the beam under the lateral load. By increasing the asymmetry, the additional axial displacement and vertical displacement increase. These additional deflections for thin beams are more than thick ones. Also, asymmetry results in increasing the natural frequencies of beams. In the free vibration analysis, the effect of asymmetry on thick beams is more than thin ones.
\end{abstract}

\section{Keywords}

cross-section asymmetry, functionally graded sandwich beams, Timoshenko beam element, bending analysis, free vibration analysis

\section{Introduction}

Sandwich beams are considered by many researchers in the civil engineering, mechanics, and aerospace industries due to their lightness and high strength. Due to the multilayer structure of these structures, they are often exposed to matrix cracks, stress concentration at the boundary of the layers, delamination, and other destructive factors, which cause the weakness and improper performance of these structures during loading. Today, researchers have been able to improve the weaknesses of sandwich structures by using functionally graded materials (FGMs). Functionally graded materials (FGMs) are a type of multilayer composite that is made from a combination of two or more materials with different volume fractions in different directions. The use of FGM materials leads to the reduction of cracks at the interface of layers in structures under high static, dynamic, and thermal loads in which the probability of cracking is high. Gradually change of the constituent materials in different directions can also eliminate residual stresses caused by differences in the thermal expansion coefficients of materials. At present, due to the widespread use of FG sandwich structures in the engineering industry, we need to analyze the free vibration, bending, and buckling behavior of these structures, accurately. Hence, a lot of research has been done on the analysis of FG sandwich beams and plates. By comparing a finite element solution and four analytical models, Apetre and Sankar [1] investigated the effects of transverse shear stress along the thickness direction of FG sandwich beams. Using the finite element method and Timoshenko beam theory, the static and dynamic behavior of the P-FGM ordinary and sandwich beam were evaluated 
by Mohanty et al. [2]. Based on the higher-order shear deformation theory, Vo et al. [3] introduced a finite element model for buckling and free vibration analysis of FG sandwich beams. Trinh et al. [4] proposed a state space approach for the free vibration analysis of FG sandwich beams with different support conditions. In order to investigate the effects of skin-core-skin thickness ratios, power-law index, length-to-span ratio, boundary conditions on natural frequencies, and buckling loads of different FG beams, a higher-order shear deformation theory for FG sandwich beam analysis was proposed by Nguyen et al. [5]. A review study was conducted by Sayyad and Ghugal [6] to analyze the problems of free vibration, buckling, and bending of FG sandwich beams under mechanical and thermal loads. Koutoati et al. [7] proposed a new finite element approach to study the effect of axial bending coupling on vibration and static behaviors of FG sandwich beams. By introducing stress equilibrium conditions, Li et al. [8] developed a new higher-order shear deformation theory. Based on this theory, he proposed a new shear deformable mixedbeam element for the analysis of FG sandwich beams.

Many researchers have studied the causes of the interaction of internal forces and moments in beams and plates. Lengyel and Németh [9] investigated the natural frequency of symmetrically cracked masonry arches. They developed an energy-based method for evaluating various effects of geometrical parameters in the free vibration analysis. Also, they obtained fundamental frequencies for monitoring the safety of structures. Rezaiee-Pajand and Gharaei-Moghaddam [10] have proposed a new element For evaluating vibration and static analysis of cracked and non-cracked non-prismatic frames. Also, Ton-That and Nguyen-Van [11] presented a combined strain element based on the first-order shear deformation theory for the study of laminated composite plate and shell.

In this paper, the governing equilibrium equations of the Timoshenko beam are used. This creates dependencies between the unknowns of the deformation field. Therefore, in addition to the simplicity of formulation, we will increase the efficiency of the new element. Several benchmark tests are used to prove the efficiency of the new element in bending and free vibration analysis. These tests evaluate the responses of the proposed element in different boundary conditions and various length to thickness ratios for different cross-sections. The results of the good elements of other researchers in each of these tests will be available for comparison. Few studies have investigated the asymmetric effect of the cross-section. In this paper, the asymmetric effect of the cross-section will be studied in both the bending and free vibration analyses. Asymmetric cross-section causes axial deformations in the beam under lateral loads. The asymmetric effect on axial deformation, deflection, and natural frequency of beam is investigated by analyzing the pinned-sliding beam and comparing its results with the pinned-pinned one.

\section{Finite element formulation}

In this section, an efficient beam element is formulated for free vibration and bending analysis of FG sandwich beams. For this purpose, the static equation governing the Timoshenko beam is used. Establishing the equilibrium equation of the beam creates dependencies between the unknowns of the field. The resulting fields, in addition to simplicity, satisfied the equilibrium equation. In this paper, the asymmetric effects of the cross-section of functionally graded sandwich beams are evaluated with the help of the proposed element.

\subsection{Calculation of shape functions}

The static equation governing Timoshenko's beam element is represented in Eq. (1). In this equation, the factors $E, G$ and $v$ represent the modulus of elasticity, the shear modulus and Poison's ratio, respectively. Also, the length of element, cross-sectional area and moment of inertia are defined by $l$, $A$ and $I$, respectively. The relationship between shear modulus and modulus of elasticity is given in Eq. (2). The shear correction coefficient is also displayed with the parameter $f_{s}$. The shear correction coefficient is related to the cross-sectional shape. It has a value of $5 / 6$ for a rectangular cross-section. The vertical displacement and rotation fields are shown with $w$ and $\phi$, respectively.

$$
\begin{aligned}
& \frac{d w}{d x}=f-\frac{1}{f_{s} G A} \frac{d}{d x}\left(E I \frac{d f}{d x}\right) l, \\
& G=\frac{E}{2(1+v)},
\end{aligned}
$$

Third-order polynomials are used for the vertical displacement field, and second-order polynomials are also used for the horizontal displacement fields and rotation fields. Fig. 1 shows the initial geometry of this proposed element. The nodal displacement vector of the initial geometry of the element is written as Eq. (3). The shape function matrix of the element is calculated by using the finite element method. The components of this matrix are listed in Appendix (A). 


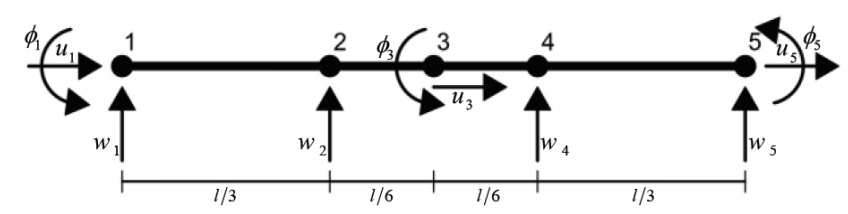

Fig. 1 Initial geometry of the proposed element

$$
\begin{aligned}
& \boldsymbol{D}_{\circ}^{\mathrm{T}}=\left[\begin{array}{llllllllll}
u_{1} & w_{1} & \phi_{1} & w_{2} & u_{3} & \phi_{3} & w_{4} & u_{5} & w_{5} & \phi_{5}
\end{array}\right]
\end{aligned}
$$

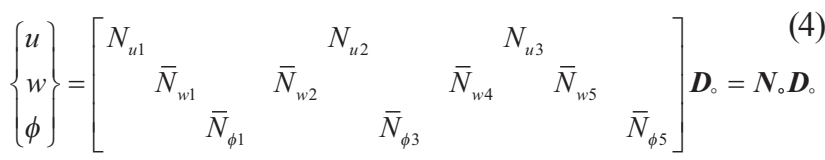

By substituting the proposed fields to equilibrium equation, Eq. (1), results in Eq. (5). Satisfying the governing equilibrium equation of the Timoshenko beam creates relationships between nodal displacements. Using these equations, degrees of freedom $w_{2}, \phi_{3}$, and $w_{4}$ will be written according to other unknowns as follows:

$$
\begin{aligned}
& {\left[\begin{array}{lll}
1 & x & x^{2}
\end{array}\right]\left(\mathrm{C}_{1}\left[\begin{array}{ll}
w_{1} & w_{5}
\end{array}\right]^{T}+\mathrm{C}_{2}\left[\begin{array}{ll}
w_{2} & w_{4}
\end{array}\right]^{T}\right)} \\
& =\left[\begin{array}{lll}
1 & x & x^{2}
\end{array}\right]\left(\mathrm{C}_{3}\left[\begin{array}{ll}
\phi_{1} & \phi_{5}
\end{array}\right]^{T}+\mathrm{C}_{4}\left[\phi_{3}\right]\right) \text {, } \\
& \mathrm{C}_{1}=\left[\begin{array}{cc}
-\frac{11}{2 l} & \frac{1}{l} \\
\frac{18}{l^{2}} & -\frac{9}{l^{2}} \\
-\frac{27}{2 l^{3}} & \frac{27}{2 l^{3}}
\end{array}\right], \mathrm{C}_{2}=\left[\begin{array}{cc}
\frac{9}{l} & -\frac{9}{2 l} \\
\frac{-45}{l^{2}} & \frac{36}{l^{2}} \\
\frac{81}{2 l^{3}} & -\frac{81}{2 l^{3}}
\end{array}\right], \\
& \mathrm{C}_{3}=\left[\begin{array}{cc}
1-\frac{4 E I}{k G A l^{2}} & -\frac{4 E I}{k G A l^{2}} \\
-\frac{3}{l} & -\frac{1}{l} \\
\frac{2}{l^{2}} & \frac{2}{l^{2}}
\end{array}\right], \mathrm{C}_{4}=\left[\begin{array}{c}
\frac{8 E I}{k G A l^{2}} \\
\frac{4}{l} \\
-\frac{4}{l^{2}}
\end{array}\right],
\end{aligned}
$$

$\left[\begin{array}{lll}w_{2} & w_{4} & \phi_{3}\end{array}\right]^{T}=\mathrm{C}_{24}{ }^{-1} \mathrm{C}_{13}\left[\begin{array}{llll}\phi_{1} & \phi_{5} & w_{1} & w_{5}\end{array}\right]^{T}$,

$$
\begin{aligned}
\mathrm{C}_{24} & =\left[\begin{array}{ccc}
\frac{9}{l} & -\frac{9}{2 l} & -\frac{8 E I}{k G A l^{2}} \\
-\frac{45}{l^{2}} & \frac{36}{l^{2}} & -\frac{4}{l} \\
\frac{81}{2 l^{3}} & -\frac{81}{2 l^{3}} & \frac{4}{l^{2}}
\end{array}\right], \\
\mathrm{C}_{13} & =\left[\begin{array}{cccc}
1-\frac{4 E I}{k G A l^{2}} & -\frac{4 E I}{k G A l^{2}} & \frac{11}{2 l} & -\frac{1}{l} \\
-\frac{3}{l} & -\frac{1}{l} & -\frac{18}{l^{2}} & \frac{9}{l^{2}} \\
\frac{2}{l^{2}} & \frac{2}{l^{2}} & \frac{27}{2 l^{3}} & -\frac{27}{2 l^{3}}
\end{array}\right] .
\end{aligned}
$$

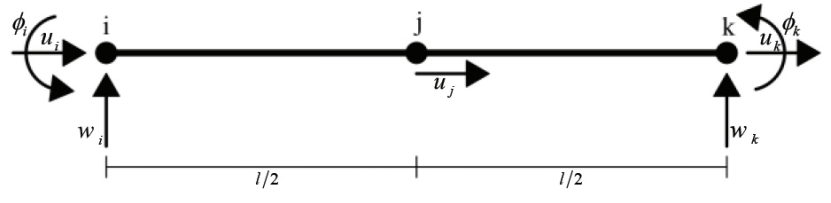

Fig. 2 The final geometry of the proposed element

The number of degrees of freedom decreases by satisfying the equilibrium equation. Reducing the number of unknowns, not only improving the efficiency of the element, but also reduces the volume of calculations.

Eliminating three degrees of freedom provides the final geometry of the proposed element in Fig. 2. The nodal displacement vector of the final proposed element is given in Eq. (10). Equation (11) shows the matrix form of the deformation fields. The shape functions of the final geometry of the element are given in Appendix (B).

$$
\boldsymbol{D}^{\mathrm{T}}=\left[\begin{array}{lllllll}
u_{i} & w_{i} & \phi_{i} & u_{j} & u_{k} & w_{k} & \phi_{k}
\end{array}\right]
$$

$$
\left\{\begin{array}{l}
u \\
w \\
\phi
\end{array}\right\}=\left[\begin{array}{ccccccc}
N_{u 1} & 0 & 0 & N_{u 2} & N_{u 3} & 0 & 0 \\
0 & N_{w 1} & N_{w 2} & 0 & 0 & N_{w 3} & N_{w 4} \\
0 & N_{\phi 1} & N_{\phi 2} & 0 & 0 & N_{\phi 3} & N_{\phi 4}
\end{array}\right] \boldsymbol{D}=N \boldsymbol{D}
$$

$\boldsymbol{N}_{u}=\left[\begin{array}{lllllll}N_{u 1} & 0 & 0 & N_{u 2} & N_{u 3} & 0 & 0\end{array}\right]$,

$\boldsymbol{N}_{w}=\left[\begin{array}{lllllll}0 & N_{w 1} & N_{w 2} & 0 & 0 & N_{w 3} & N_{w 4}\end{array}\right]$,

$\boldsymbol{N}_{\phi}=\left[\begin{array}{lllllll}0 & N_{\phi 1} & N_{\phi 2} & 0 & 0 & N_{\phi 3} & N_{\phi 4}\end{array}\right]$.

\subsection{Discretization of the governing equation of free} vibration and bending analysis

In FGM beams, the change of material characteristics such as modulus of elasticity, mass density, and shear modulus is assumed based on the exponential relation proportional to. In Eq. (13), defines the homogenized equivalent factor for the factors of the primary constituent materials $P_{c}$ (ceramic) and $P_{m}$ (metal). The $V_{c}$ function is the volume fraction of a ceramic material that can vary along the thickness direction $\left(0 \leq V_{c} \leq 1\right)$. Equations (14) shows the distribution of the $V_{c}$ function for each type of cross-sections that are used in this paper. The parameter $\mathrm{h}$ represents the thickness of beam.

$P(z)=\left(P_{c}-P_{m}\right) V_{C}(z)+P_{m}$

Type $A: \quad V_{c}(z)=\left(\frac{1}{2}-\frac{z}{h}\right)^{k} \quad z_{1} \leq z \leq z_{4}$

$$
\text { Type } B:\left\{\begin{array}{lll}
\text { Bottom FGM layer: } & V_{c}(z)=\left(\frac{z-z_{1}}{z_{2}-z_{1}}\right)^{k} & z_{1} \leq z \leq z_{2} \\
\text { Core ceramic layer: } & V_{c}(z)=1 & z_{2} \leq z \leq z_{3} \\
\text { Top FGM layer: } & V_{c}(z)=\left(\frac{z-z_{4}}{z_{3}-z_{4}}\right)^{k} & z_{3} \leq z \leq z_{4}
\end{array}\right.
$$


(16)

Type $: \begin{cases}\text { Bottom ceramic layer: } V_{c}(z)=1 & z_{1} \leq z \leq z_{2} \\ \text { Core FGM layer: } \quad V_{c}(z)=\left(\frac{z-z_{3}}{z_{2}-z_{3}}\right)^{k} \quad z_{2} \leq z \leq z_{3} \\ \text { Top metal layer: } \quad V_{c}(z)=0 \quad z_{3} \leq z \leq z_{4}\end{cases}$

The cross-section $A$ is considered as an FGM layer. The cross-section $B$ has a ceramic core enclosed in two FGM layers. Also, the cross-section of type $C$ consists of an FGM core and homogeneous ceramic and metal layers at the bottom and top of the core, respectively. Fig. 3 shows the variation of the materials in the cross-sections $A, B(1-2-1)$ and $C(1-2-1)$.

By utilizing the shape functions, the horizontal and vertical displacement fields, and rotation functions are written according to the nodal displacements vector. The matrix form of the strain energy function of the element which is calculated based on the shape functions is given in Eq. (17). In this equation, the normal and shear stresses are represented by the parameters $\sigma_{x x}$ and $\tau_{x z}$, respectively, and the normal and shear strains are also represented by the parameters $\varepsilon_{x x}$ and $\gamma_{x z}$. The relationship between stress and strain is shown in Eq. (18). By substituting the stress and strain vectors in Eq. (17), the matrix form of strain energy for the element will be available as Eq. (19). The stiffness matrix is calculated by minimizing the strain energy function in terms of the nodal displacement vector. The longitudinal displacement field is shown with $u$.

$$
\begin{aligned}
& U=\frac{1}{2} \int_{0}^{l} \int_{A}\left(\sigma_{x x} \varepsilon_{x x}+\tau_{x z} \gamma_{x z}\right) d A d x \\
& \sigma_{x x}=E \varepsilon_{x x}, \tau_{x z}=f_{s} G \gamma_{x z} \\
& U=\int_{0}^{l}\left[\begin{array}{l}
A_{0}\left(\frac{\partial u}{\partial x}\right)^{2}-2 A_{1}\left(\frac{\partial u}{\partial x}\right)\left(\frac{\partial \phi}{\partial x}\right) \\
\left.+A_{2}\left(\frac{\partial \phi}{\partial x}\right)^{2}+B_{0}\left(\begin{array}{l}
\left(\frac{\partial w}{\partial x}\right)^{2} \\
-2 \frac{\partial w}{\partial x} \phi+(\phi)^{2}
\end{array}\right)\right] d x
\end{array}\right]
\end{aligned}
$$

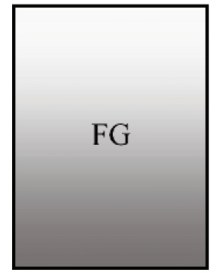

Section A
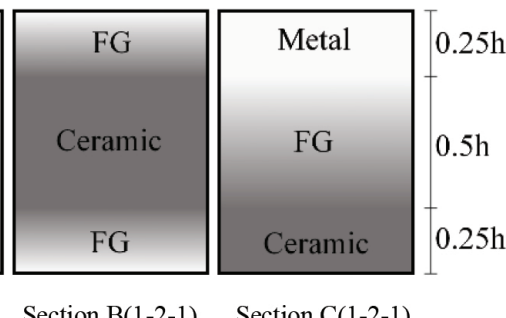

Fig. 3 The variation of the materials in the cross-section

$$
\begin{aligned}
& {\left[A_{0}, A_{l}, A_{2}\right]=\int_{A} E_{e}\left[1, z, z^{2}\right] d A, \quad B_{o}=\int_{A} f_{s} G_{e} d A} \\
& \boldsymbol{K}=\int_{0}^{l}\left[\begin{array}{l}
A_{0} \boldsymbol{N}_{u, x}^{T} \boldsymbol{N}_{u, x}-A_{1} \boldsymbol{N}_{u, x}^{T} \boldsymbol{N}_{\phi, x}-A_{1} \boldsymbol{N}_{\phi, x}^{T} \boldsymbol{N}_{u, x} \\
\left.+A_{2} \boldsymbol{N}_{\phi, x}^{T} \boldsymbol{N}_{\phi, x}+B_{0}\left(\begin{array}{c}
\boldsymbol{N}_{w, x}^{T} \boldsymbol{N}_{w, x}-\boldsymbol{N}_{w, x}^{T} \boldsymbol{N}_{\phi} \\
-\boldsymbol{N}_{\phi}^{T} \boldsymbol{N}_{w, x}+\boldsymbol{N}_{\phi}^{T} \boldsymbol{N}_{\phi}
\end{array}\right)\right] d x
\end{array}\right.
\end{aligned}
$$

The matrix form of the kinetic energy function of the element is also given in Eq. (22). First, by integrating on the cross-section, the kinetic energy function is simplified. Then by substituting the displacement and rotation fields and based on Hamilton's principle, the element mass matrix is written as Eq. (24). The factor $\rho$ indicates the mass density.

$$
\begin{aligned}
& T=\frac{1}{2} \int_{0}^{l} \int_{A} \rho\left(\left(\frac{\partial u}{\partial t}-z \frac{\partial \phi}{\partial t}\right)^{2}+\left(\frac{\partial w}{\partial t}\right)^{2}\right) d A d x \\
& =\frac{1}{2} \int_{0}^{l}\left[\begin{array}{l}
I_{0}\left(\frac{\partial u}{\partial t}\right)^{2}-2 I_{1}\left(\frac{\partial u}{\partial t}\right)\left(\frac{\partial \phi}{\partial t}\right) \\
+I_{2}\left(\frac{\partial \phi}{\partial t}\right)^{2}+I_{0}\left(\frac{\partial w}{\partial t}\right)^{2}
\end{array}\right] d x \\
& {\left[I_{0}, I_{1}, I_{2}\right]=\int \rho_{e}\left[1, z, z^{2}\right] d A} \\
& \boldsymbol{M}=\int_{0}^{l}\left[\begin{array}{l}
I_{0} \boldsymbol{N}_{u}^{T} \boldsymbol{N}_{u}-I_{1} \boldsymbol{N}_{u}^{T} \boldsymbol{N}_{\phi}-I_{1} \boldsymbol{N}_{\phi}^{T} \boldsymbol{N}_{u} \\
+I_{2} \boldsymbol{N}_{\phi}^{T} \boldsymbol{N}_{\phi}+I_{0} \boldsymbol{N}_{w}^{T} \boldsymbol{N}_{w}
\end{array}\right] d x
\end{aligned}
$$

Finally, the governing equation of the element is made available in Eq. (25). The stiffness and mass matrices are represented by $\boldsymbol{K}$ and $\boldsymbol{M}$, respectively. Also, the nodal force vector is shown with $\boldsymbol{F}$.

$M \ddot{D}+K D=F$

\section{Numerical tests}

In FGM structures, asymmetry in the cross-section causes additional axial deformations compared to the state of symmetry in the cross-section. Usually, research into the construction of new elements for bending and free vibration analysis focuses only on pure bending, and studies on cross-section asymmetry are very limited. In the present study, in addition to pure bending, the asymmetric effects of the cross-section on the longitudinal and transverse deformations of the beam for free vibration and bending analysis will also be investigated.

A large number of benchmark tests are used to demonstrate the capabilities of the new element in free vibration and the bending analysis of FG sandwich beams. In these tests, different boundary conditions, including 
clamped (C), the free end $(\mathrm{F})$, pinned with free axial displacement $(\mathrm{P})$, and pinned without axial displacement $(\mathrm{S})$ are used. Aluminum $(m)$ and alumina $(c)$ are the primary constituent materials of beams in this paper. The characteristics of these materials are available in Table 1. The good elements responses of other researchers in each benchmark are presented for comparison.

The elements used in this paper are as follows:

1. Displacement beam element based on first-order shear deformation theory; DFS [8]

2. Mixed beam element based on first-order shear deformation theory; MFS [8]

3. Beam element based on first-order shear deformation theory; FOBT [12]

\subsection{Bending analysis}

\subsubsection{Convergence}

First, the convergence of the proposed element in the bending analysis of the FGM beam is examined. Fig. 4 examines the convergence of the displacement responses of the free end of the cantilever beam with a length of one meter, a thickness of $200 \mathrm{~mm}$, and a width of $50 \mathrm{~mm}$ under a concentrated load of $500 \mathrm{KN}$ at the tip of the beam. Crosssections $B$ and $C$ with distribution (3-4-3) are used. In this distribution, the thickness of the upper and lower layers is equal to and the core has a thickness of $80 \mathrm{~mm}$. Also, $k=5$ is considered. The proposed element gives an accurate answer when using the symmetric cross-section $B$, even with two elements. Also, in the case of using asymmetric cross-section, the response of proposed element has high accuracy and rapid convergence, even in the coarse mesh. The percentage error of proposed element even for the coarse mesh with only two elements for the cross-sections $B$ (3-4-3) and $C(3-4-3)$ is $0 \%$ and $0.25 \%$, respectively. Also, using a four-element mesh, the percentage error of proposed element for cross-section $C$ (3-4-3) will be about $0.08 \%$. So, the proposed element has a rapid convergence in static problems.

\subsubsection{Validation}

The maximum displacement of the beam with a width of and a thickness of $20 \mathrm{~cm}$ under different support conditions is given in Table 2. In the boundary conditions of the pinned-sliding (S-P) and the clamped-clamped (C-C), the two-meter-long beam is subjected to a uniformly distributed load of $5 \mathrm{MN} / \mathrm{m}$. Also, in the case of clamped-free supports (C-F), a concentrated load of $5 \mathrm{MN}$ applies to the tip of the beam with a length of one meter. The obtained
Table 1 Characteristics of the constituent materials of the beam

\begin{tabular}{lccc}
\hline Material & $E(\mathrm{Gpa})$ & $\rho\left(\mathrm{kg} / \mathrm{m}^{3}\right)$ & $v$ \\
\hline $\mathrm{Al}(m)$ & 70 & 2702 & 0.3 \\
$\mathrm{Al}_{2} \mathrm{O}_{3}$ & 380 & 3960 & 0.3 \\
\hline
\end{tabular}
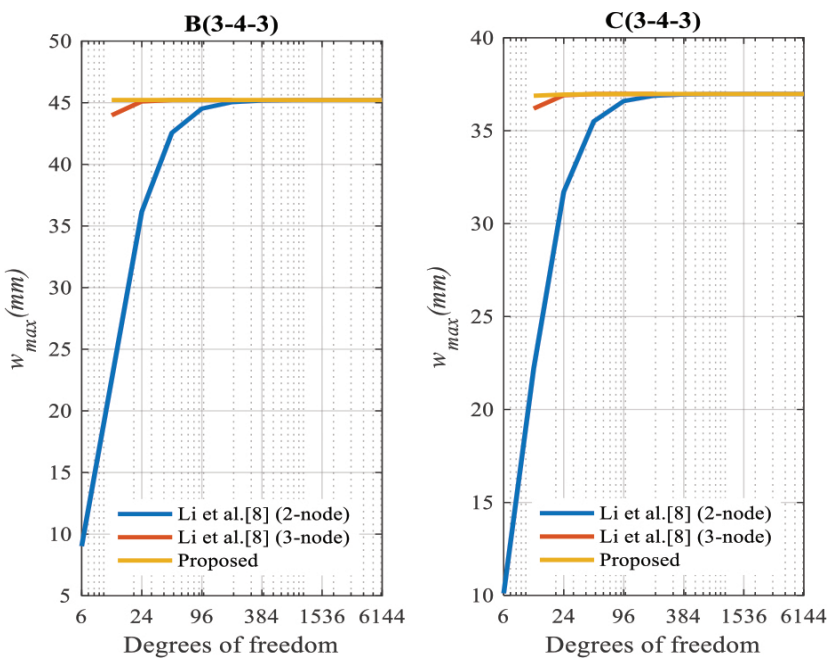

Fig. 4 The maximum displacement $w_{\max }$ of the clamped beam under concentrated load on the tip

results are compared with those of Li et al. [8] in Table 2. This Table reveals that the proposed element has high accuracy in bending analysis for different cross-sections and under different boundary conditions.

\subsubsection{Asymmetric effects of the cross-section}

After the validation of the proposed element, the asymmetric effect of the cross-section in bending analysis is investigated. For this purpose, symmetrical cross-section $B$ (1-2-1) and asymmetric cross-section $C$ (1-2-1) are considered. For the beam with symmetrical cross-section, there is no axial deformation in the beam under lateral load. But, using the asymmetrical cross-section, results in axial displacement in the beam even under lateral forces. To study the effects of asymmetry, the pinned-sliding beam (S-P) under a uniformly distributed load of $5 \mathrm{MN} / \mathrm{mis}$ considered. The length, width, and thickness of the beam are equal to $2 \mathrm{~m}, 5 \mathrm{~cm}$, and $20 \mathrm{~cm}$, respectively. The axial displacement of this beam with asymmetric cross-section $C$ (1-2-1) under the lateral load mentioned above is shown in Fig. 5. Changing the distribution of the material properties causes the shifting of the normal center of the cross-section from its geometric center. The longitudinal displacements of the kinetic center alongside those of the geometric center are came in Fig. 5. Due to this figure, the use of an asymmetric cross-section leads to additional horizontal displacement that does not occur for a symmetrical cross-section. 
Table 2 Investigating the maximum displacement $w_{\max }(\mathrm{mm})$ of the beam in different boundary conditions

\begin{tabular}{lcccccccccc}
\hline Type & $k$ & & C-C & & \multicolumn{3}{c}{ S-P } & & \multicolumn{3}{c}{ C-F } \\
& & Proposed & DFS [8] & MFS[8] & Proposed & DFS[8] & MFS[8] & Proposed & DFS[8] & MFS[8] \\
\hline A & 0 & 18.50 & 18.50 & 18.50 & 84.25 & 84.29 & 84.29 & 13.57 & 13.57 & 13.57 \\
& 0.5 & 28.19 & 28.19 & 28.15 & 129.62 & 129.68 & 129.64 & 20.86 & 20.86 & 20.85 \\
& 1 & 36.46 & 36.46 & 36.48 & 168.37 & 168.45 & 168.47 & 27.08 & 27.09 & 27.09 \\
& 5 & 56.42 & 56.42 & 57.89 & 256.30 & 256.43 & 257.90 & 41.29 & 41.29 & 41.58 \\
& 10 & 62.83 & 62.84 & 64.50 & 282.30 & 282.43 & 284.09 & 45.49 & 45.51 & 45.84 \\
B (3-4-3) & 0 & 18.50 & 18.50 & 18.50 & 84.25 & 84.29 & 84.29 & 13.57 & 13.57 & 13.57 \\
& 0.5 & 27.31 & 27.31 & 27.15 & 126.70 & 126.75 & 126.59 & 20.38 & 20.38 & 20.35 \\
& 1 & 34.63 & 34.63 & 34.37 & 162.19 & 162.27 & 162.01 & 26.07 & 26.07 & 26.02 \\
& 5 & 59.12 & 59.13 & 58.68 & 281.62 & 281.76 & 281.32 & 45.22 & 45.22 & 45.13 \\
C (3-4-3) & 10 & 66.06 & 66.06 & 65.73 & 315.36 & 315.52 & 315.19 & 50.63 & 50.63 & 50.57 \\
& 0 & 35.49 & 35.51 & 35.43 & 166.57 & 166.66 & 166.58 & 26.77 & 26.77 & 26.76 \\
& 0.5 & 41.08 & 41.11 & 41.09 & 192.76 & 192.85 & 192.84 & 30.98 & 30.98 & 30.98 \\
& 1 & 44.22 & 44.26 & 44.49 & 207.29 & 207.41 & 207.64 & 33.32 & 33.32 & 33.37 \\
& 5 & 49.35 & 49.41 & 51.38 & 229.92 & 230.04 & 232.02 & 36.98 & 36.98 & 37.37 \\
& 10 & 49.92 & 49.98 & 52.67 & 231.88 & 232.00 & 234.69 & 37.30 & 37.30 & 37.84 \\
\hline
\end{tabular}

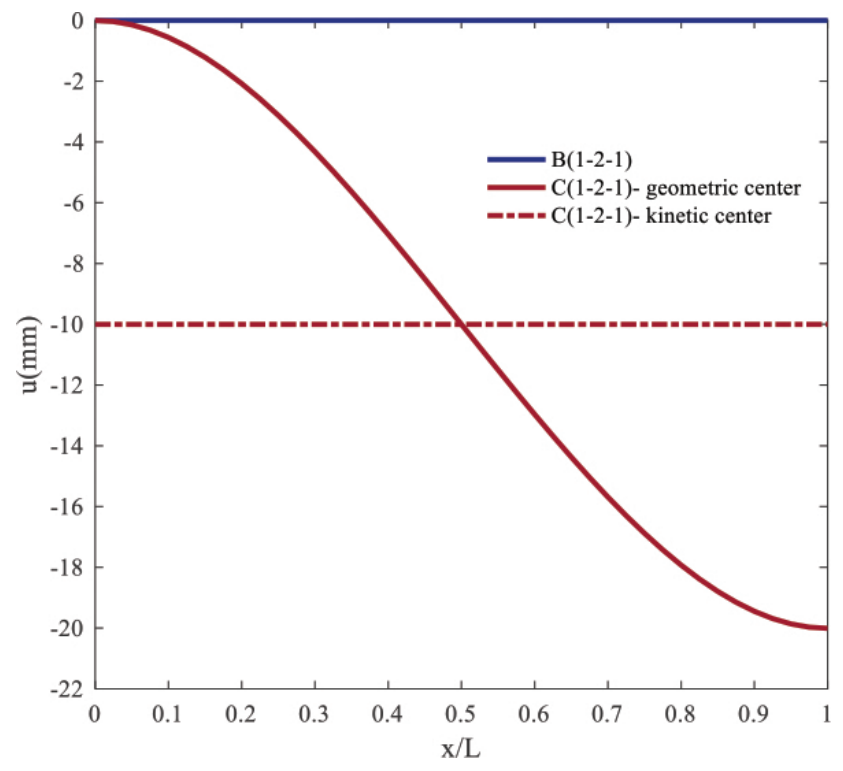

Fig. 5 Horizontal displacement $(u)$ along the pinned-sliding beam with symmetrical and asymmetrical cross-sections under a uniformly distributed load

By utilizing the simple-simple support conditions, as shown in Fig. 6(a), the axial deformation of the beam with cross-section $C$ (1-2-1) will be greatly reduced. Also, Fig. 6(b), and Fig. 6(c) show the changes of rotation and vertical deflection values along the beam with an asymmetric cross-section of $C$ (1-2-1) for both of (S-S) and (S-P) supports conditions.

By increasing the value of the parameter $k$ in crosssection $C$ (1-2-1), the maximum horizontal displacement of the pinned-sliding beam increases under a uniformly distributed load. The maximum non-dimensional horizontal displacement of the pinned-sliding beam for different values of $k$ under a uniformly distributed load is shown in Fig. 7. For any value of $k$, division of the maximum horizontal displacement of the beam by the maximum horizontal displacement of the same beam with $k=0$ determines the amount of the maximum non-dimensional horizontal displacement. It should be noted that the normalized longitudinal displacements of the kinetic center are equal to the maximum normalized longitudinal displacements of the geometric center.

As the value of $k$ rises in cross-section $C(1-2-1)$, the rate of asymmetric increases. Also, changing the value of $k$ changes the moment of inertia. To evaluate the asymmetric effect of the cross-section independent of the moment of inertia, Fig. 8 shows the ratios of the beam's maximum deflections with a cross-section of $C(1-2-1)$ in the sliding-pinned support condition $\left(w_{S-P}\right)_{\max }$ to its values for the simple-simple support condition $\left(w_{S-S}\right)_{\max }$ for length-tothickness ratios 5, 10, 100, and 1000 under the uniform distribution load.

The effect of the length-to-thickness ratio on the vertical deflection of the beam has also shown in Fig. 8. Base on this figure, it can be deduced that by increasing the aspect ratio of the beam, additional vertical deflection due to asymmetry of the cross section increases. For $k=6$, the ratio of $\left(w_{S-P}\right)_{\max }$ to $\left(w_{S-S}\right)_{\max }$ for the values of $\mathrm{L} / \mathrm{h}$ equal to 5, 10, 100 and 1000 are 1.3654, 1.3958, 1.4069 and 1.4070, respectively. It should be noted that the change of the 


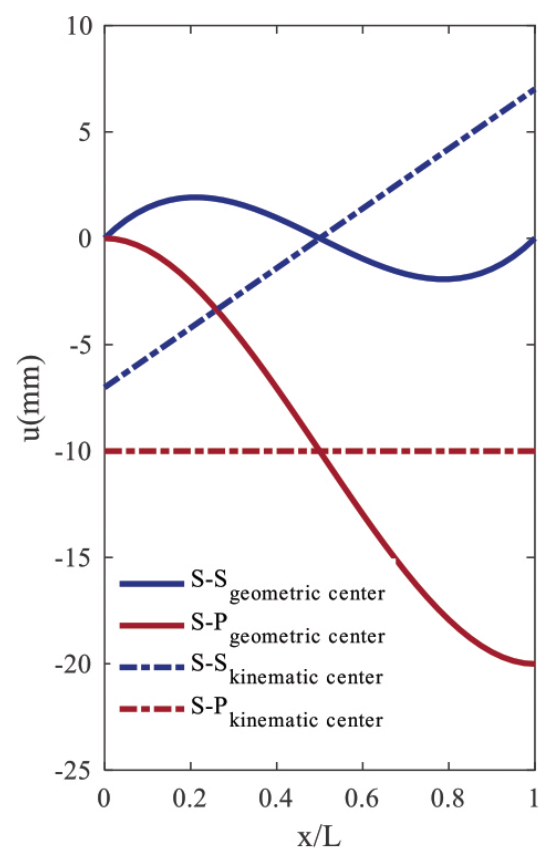

(a)

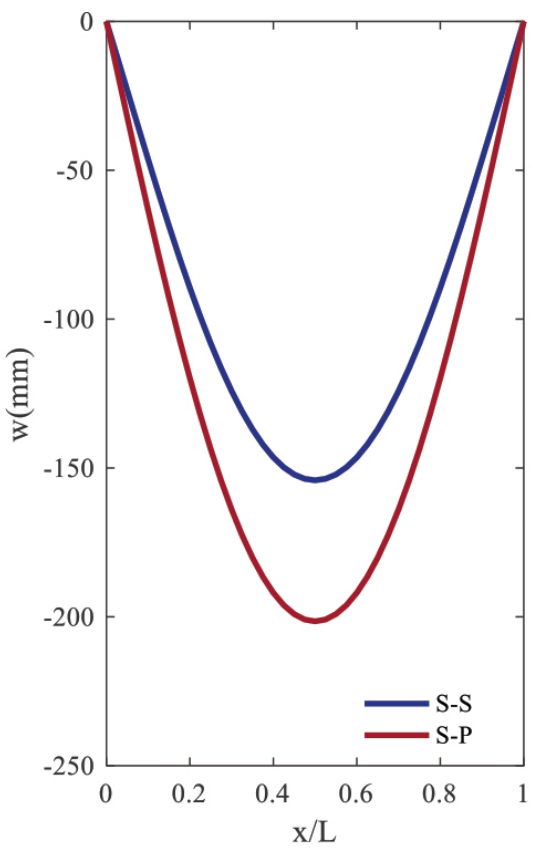

(b)

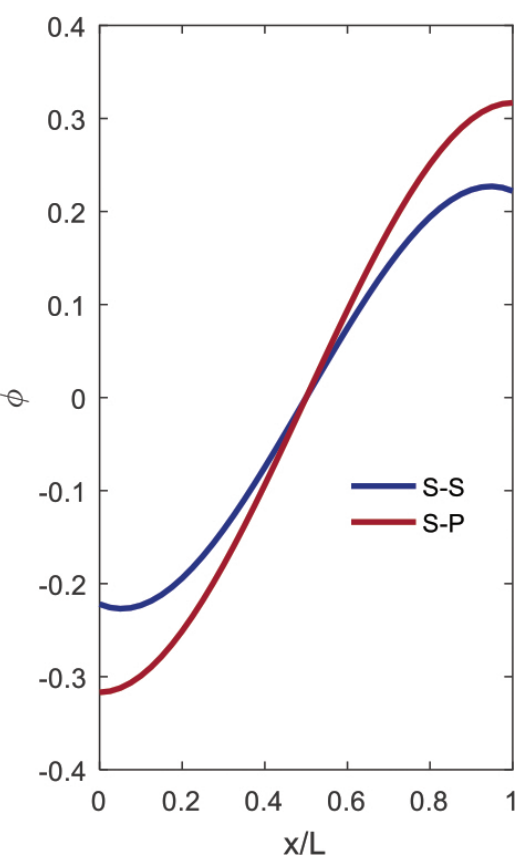

(c)

Fig. 6 Horizontal displacement $u$ (a), rotation $\phi$ (b), and vertical deflection $w$ (c) along the beam with cross-section C (1-2-1) with support conditions S-S and S-P under a uniformly distributed load

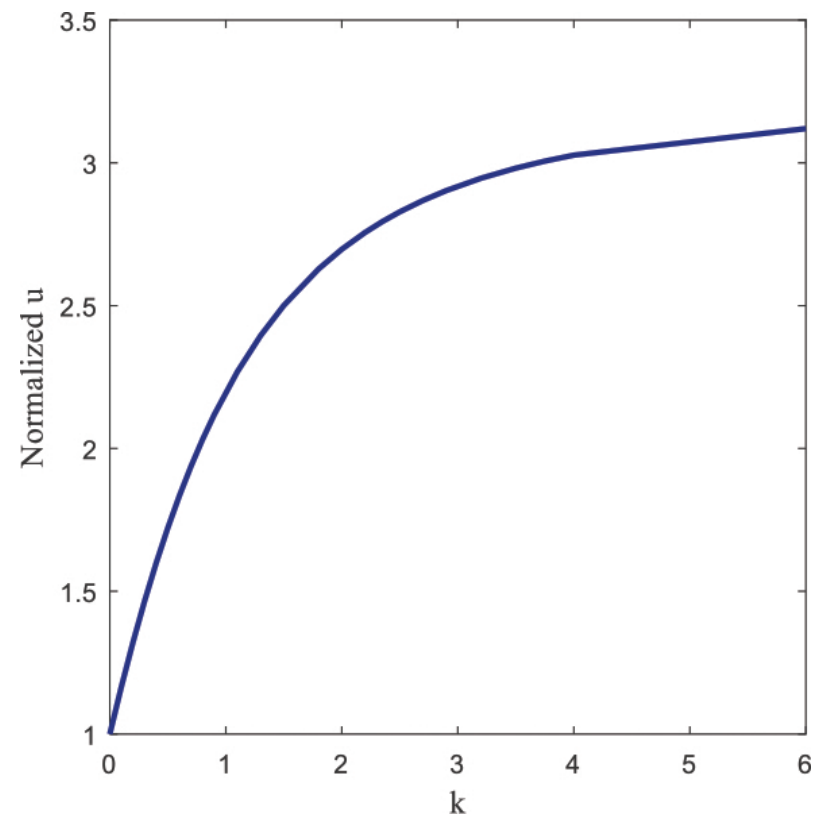

Fig. 7 The maximum non-dimensional horizontal displacement of the pinned-sliding beam with cross-section $C$ (1-2-1) under a uniformly distributed load

$\left(w_{S-P}\right)_{\max } /\left(w_{S-S}\right)_{\max }$ parameter by increasing the aspect ratio from 10 to 1000 is only equal to 0.0112 while this change will be equal to 0.0304 when the $\mathbf{L} / h$ parameter increases from 5 to 10 .

As the $k$ parameter rises, the ratio of $\left(w_{S-P}\right)_{\max }$ to $\left(w_{S-S}\right)_{\max }$ increases. The rate of this increase is greater for small values of $k$. The use of an asymmetric cross-section for beams

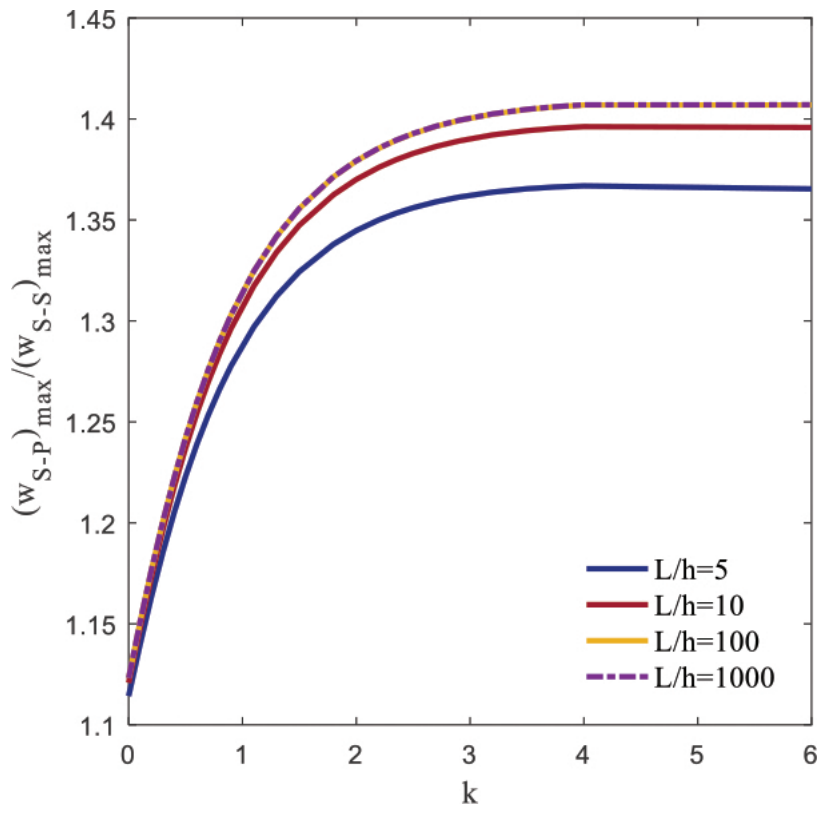

Fig. 8 Ratio of the beam's maximum deflection in pinned-sliding support condition to simple-simple support condition for the crosssection of $C(1-2-1)$

with simple-simple support conditions under lateral load will result in a non-zero support reaction in the direction of the horizon. Fig. 9 shows the non-dimensional horizontal support reaction of the simple-simple beam with cross-section $C$ (1-2-1) for different ratios of the elastic modulus under a uniformly distributed load. The elastic modulus of the upper and lower layers is defined by $E_{u}$ and $E_{l}$, 


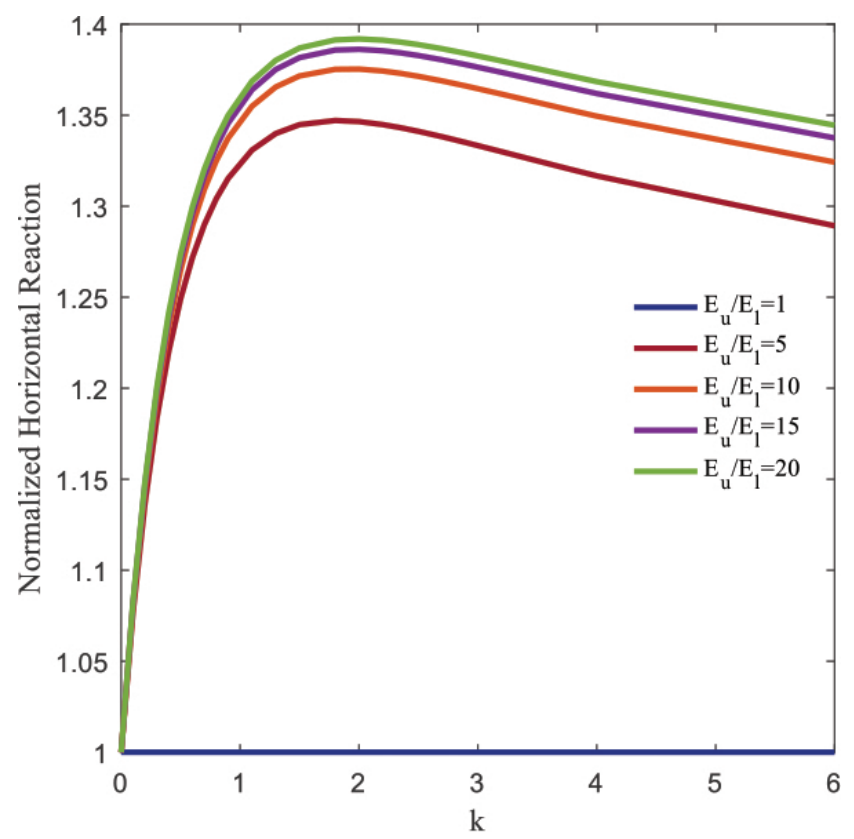

Fig. 9 Non-dimensional horizontal support reaction of the simple-simple beam with cross-section $C$ (1-2-1) under a uniformly distributed load

respectively. For each value of $k$, the non-dimensional horizontal support reaction is obtained by dividing the value of the horizontal support reaction for $k$ by its value at $k=0$. For higher elastic modulus ratios, the amount of horizontal force produced at the support is larger.

Fig. 10 shows the effect of the parameter $k$ as well as the ratio of modulus of elasticity $E_{u} / E_{l}$ on the deflection of the beam with the cross-section $C(1-2-1)$. The beam has a length-to-thickness ratio of 5. Based on this figure, increasing the parameters $k$ and $E_{u} / E_{l}$ increases the $\left(w_{S-P}\right)_{\max }$ to $\left(w_{S-S}\right)_{\max }$ ratio.

\subsection{Free vibration analysis}

In this section, the efficiency of the proposed element in the free vibration analysis is examined in comparison with the good elements of other researchers. For the study, two length-to-thickness ratios of 5 and 20 are exploited. Also, different boundary conditions are assumed in this study. First, the convergence of the proposed element is studied in examining the results of free vibration analysis. Fig. 11 makes the convergence of the proposed element available in clamped support conditions for cross-sections $A, B$, and $C$. In this study, the non-dimensional frequency $\bar{\omega}$ is used for comparison. This parameter is available in Eq. (26). $E_{m}$ and $\rho_{m}$, are modulus of elasticity and density of metal, respectively. Also, the length and thickness of beam are shown with $L$ and $h$, respectively.

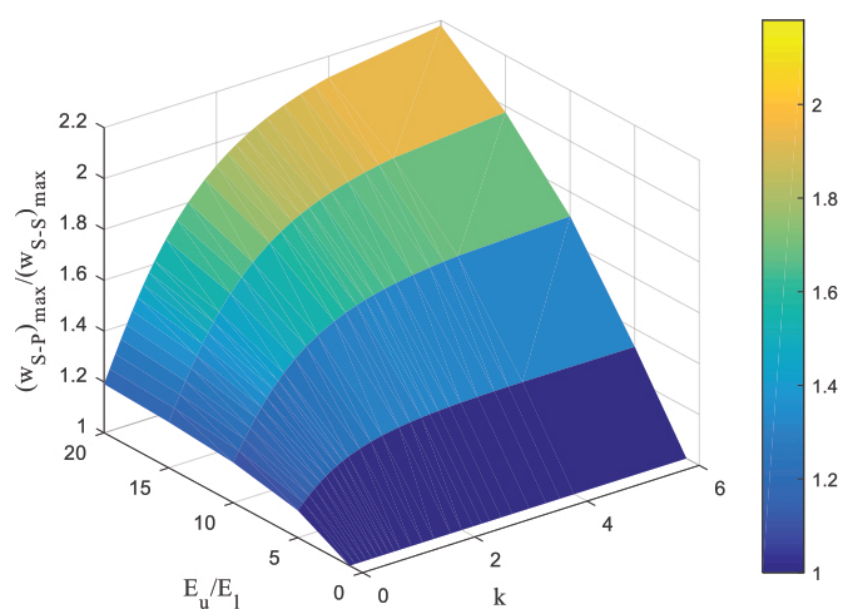

Fig. 10 Ratio of to for the beam with cross-section $C$ (1-2-1)

$\bar{\omega}=\frac{\omega L}{h} \sqrt{\frac{\rho_{m}}{E_{m}}}$

Fig. 11 demonstrates the rapid convergence and high accuracy of the proposed element response even in the coarse mesh. Compared to the fine mesh response, not all the frequency results of proposed element in Fig. 11 even for coarse mesh with only two elements have percentage error greater than $4.5 \%, 4.1 \%$ and $7.2 \%$ for sections $A, B$ (1-2-1) and $C(1-2-1)$, respectively. Also, by increasing the number of element to 4 , these percentage errors become less than $1.1 \%, 0.94 \%$ and $1.92 \%$ for cross-sections $A, B(1-2-1)$ and $C(1-2-1)$, respectively.

In the following, the results of free vibration analysis of the proposed element are compared with the good elements of other researchers. Table 3 shows the results of free vibration analysis in twenty-element mesh for beams with length-to-thickness ratios of 20 and 5 for the proposed element. In this study, cross-section $A$ was considered for the beam. The results of the good elements of other researchers are also presented in this table.

Table 4 presents the non-dimensional frequency responses of the beam with cross-sections $B$ (1-2-1) and $B$ (2-2-1) for the length to thickness ratios 20 and 5. Also, the results of free vibration analysis of the beam with cross-sections $C(1-2-1)$ and $C(2-2-1)$ are shown in Table 5. For comparison, the non-dimensional frequency responses $\bar{\omega}$ of other researchers are available in each of these tables.

The phrase (2-2-1) in the section name indicates that the thickness of the upper layer is equal to $40 \%$ of the total thickness of the beam, and the thickness of the lower layer is equal to $20 \%$ of the total thickness of the beam. 

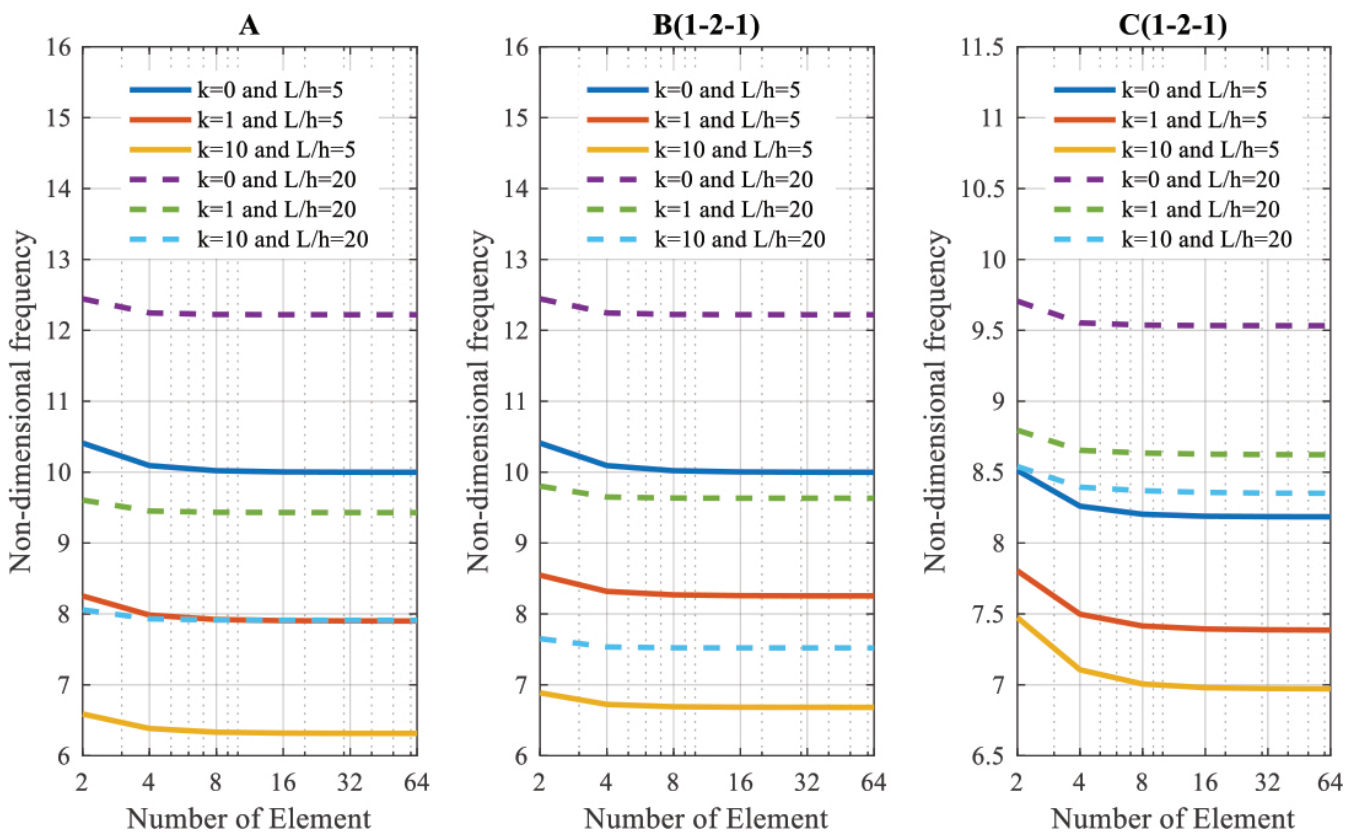

Fig. 11 Investigation of the non-dimensional frequency convergence of the proposed element with clamped support condition for cross-sections $A, B(1-2-1)$ and $C(1-2-1)$.

Table 3 Non-dimensional frequency $\bar{\omega}$ in different boundary conditions for the beam with cross-section $A$

\begin{tabular}{|c|c|c|c|c|c|c|c|c|c|c|c|c|}
\hline \multirow{2}{*}{$\mathrm{k}$} & \multirow{2}{*}{\multicolumn{2}{|c|}{ Theory }} & \multicolumn{5}{|c|}{$L / h=20$} & \multicolumn{5}{|c|}{$L / h=5$} \\
\hline & & & $\mathrm{C}-\mathrm{C}$ & $\mathrm{C}-\mathrm{S}$ & C-P & $\mathrm{C}-\mathrm{F}$ & S-P & $\mathrm{CC}$ & $\mathrm{C}-\mathrm{S}$ & C-P & $\mathrm{C}-\mathrm{F}$ & S-P \\
\hline \multirow[t]{3}{*}{0} & FOBT & Trinh et al. [4] & 12.221 & 8.482 & 8.482 & 1.950 & 5.460 & - & - & - & - & - \\
\hline & FOBT & Şimşek [12] & 12.224 & - & - & 1.950 & - & 10.034 & - & - & 1.895 & 5.152 \\
\hline & & Proposed & 12.221 & 8.481 & 8.481 & 1.950 & 5.460 & 10.001 & 7.467 & 7.467 & 1.894 & 5.153 \\
\hline \multirow[t]{3}{*}{1} & FOBT & Trinh et al. [4] & 9.430 & 6.619 & 6.538 & 1.501 & 4.204 & - & - & - & - & - \\
\hline & FOBT & Şimşek [12] & 9.431 & - & - & 1.501 & - & 7.925 & - & - & 1.463 & 3.990 \\
\hline & & Proposed & 9.430 & 6.619 & 6.538 & 1.501 & 4.204 & 7.903 & 5.906 & 5.841 & 1.463 & 3.971 \\
\hline \multirow[t]{3}{*}{10} & FOBT & Trinh et al. [4] & 7.910 & 5.550 & 5.496 & 1.265 & 3.540 & - & - & - & - & - \\
\hline & FOBT & Şimşek [12] & 7.913 & - & - & 1.265 & - & 6.341 & - & - & 1.224 & 3.313 \\
\hline & & Proposed & 7.911 & 5.550 & 5.496 & 1.265 & 3.540 & 6.318 & 4.798 & 4.756 & 1.224 & 3.296 \\
\hline
\end{tabular}

Table 4 Non-dimensional frequency $\bar{\omega}$ in different boundary conditions for the beam with section $B$

\begin{tabular}{|c|c|c|c|c|c|c|c|c|c|c|c|}
\hline \multirow{3}{*}{$\begin{array}{l}\begin{array}{l}\text { Cross } \\
\text { section }\end{array} \\
B(1-2-1)\end{array}$} & \multirow{3}{*}{$\frac{\mathrm{k}}{\mathrm{k}}$} & \multirow{2}{*}{\multicolumn{2}{|c|}{ Models }} & \multicolumn{4}{|c|}{$L / h=20$} & \multicolumn{4}{|c|}{$L / h=5$} \\
\hline & & & & \multirow{2}{*}{$\frac{\mathrm{SP}}{5.461}$} & \multirow{2}{*}{$\frac{\mathrm{CC}}{12.221}$} & \multirow{2}{*}{$\frac{\mathrm{CF}}{1.950}$} & \multirow{2}{*}{$\frac{\mathrm{CP}}{8.482}$} & \multirow{2}{*}{$\frac{\mathrm{SP}}{5.153}$} & \multirow{2}{*}{$\frac{\mathrm{CC}}{9.998}$} & \multirow{2}{*}{$\frac{\mathrm{CF}}{1.895}$} & \multirow{2}{*}{$\frac{\mathrm{CP}}{7.465}$} \\
\hline & & FOBT & Vo et al. [3] & & & & & & & & \\
\hline & & & Proposed & 5.460 & 12.221 & 1.950 & 8.481 & 5.153 & 10.001 & 1.894 & 7.467 \\
\hline & 1 & FOBT & Vo et al. [3] & 4.289 & 9.632 & 1.531 & 6.672 & 4.100 & 8.255 & 1.497 & 6.053 \\
\hline & & & Proposed & 4.288 & 9.632 & 1.530 & 6.671 & 4.100 & 8.257 & 1.497 & 6.054 \\
\hline & 10 & FOBT & Vo et al. [3] & 3.340 & 7.521 & 1.192 & 5.202 & 3.224 & 6.682 & 1.171 & 4.828 \\
\hline & & & Proposed & 3.340 & 7.521 & 1.191 & 5.202 & 3.223 & 6.683 & 1.171 & 4.828 \\
\hline \multirow[t]{6}{*}{$B(2-2-1)$} & 0 & FOBT & Vo et al. [3] & 5.461 & 12.221 & 1.950 & 8.482 & 5.153 & 9.998 & 1.895 & 7.465 \\
\hline & & & Proposed & 5.460 & 12.221 & 1.950 & 8.481 & 5.153 & 10.001 & 1.894 & 7.467 \\
\hline & 1 & FOBT & Vo et al. [3] & 4.160 & 9.345 & 1.485 & 6.472 & 3.979 & 8.033 & 1.453 & 5.883 \\
\hline & & & Proposed & 4.159 & 9.345 & 1.484 & 6.472 & 3.979 & 8.035 & 1.453 & 5.884 \\
\hline & 10 & FOBT & Vo et al. [3] & 3.161 & 7.119 & 1.128 & 4.924 & 3.046 & 6.332 & 1.108 & 4.571 \\
\hline & & & Proposed & 3.160 & 7.118 & 1.127 & 4.923 & 3.046 & 6.334 & 1.108 & 4.572 \\
\hline
\end{tabular}


Table 5 Non-dimensional frequency in $\bar{\omega}$ different boundary conditions for the beam with cross-section $C$

\begin{tabular}{|c|c|c|c|c|c|c|c|c|c|c|c|}
\hline \multirow{2}{*}{$\begin{array}{l}\text { Cross- } \\
\text { section }\end{array}$} & \multirow{2}{*}{$k$} & \multirow{2}{*}{\multicolumn{2}{|c|}{ Models }} & \multicolumn{4}{|c|}{$L / h=20$} & \multicolumn{4}{|c|}{$L / h=5$} \\
\hline & & & & SP & $\mathrm{CC}$ & $\mathrm{CF}$ & $\mathrm{CP}$ & SP & $\mathrm{CC}$ & $\mathrm{CF}$ & $\mathrm{CP}$ \\
\hline \multirow[t]{6}{*}{$C(1-2-1)$} & 0 & FOBT & Vo et al. [3] & 4.243 & 9.533 & 1.515 & 6.603 & 4.043 & 8.185 & 1.481 & 5.995 \\
\hline & & & Proposed & 4.243 & 9.534 & 1.514 & 6.603 & 4.043 & 8.187 & 1.481 & 5.996 \\
\hline & 1 & FOBT & Vo et al. [3] & 3.837 & 8.623 & 1.370 & 5.973 & 3.627 & 7.386 & 1.339 & $5.40 \mathrm{~S}$ \\
\hline & & & Proposed & 3.836 & 8.626 & 1.370 & 5.973 & 3.628 & 7.391 & 1.338 & 5.410 \\
\hline & 10 & FOBT & Vo et al. [3] & 3.721 & 8.351 & 1.330 & 5.790 & 3.471 & 6.972 & 1.293 & 5.151 \\
\hline & & & Proposed & 3.721 & 8.355 & 1.330 & 5.791 & 3.471 & 6.977 & 1.293 & 5.154 \\
\hline \multirow[t]{6}{*}{$C(2-2-1)$} & 0 & FOBT & Vo et al. [3] & 3.811 & 8.572 & 1.361 & 5.935 & 3.622 & 7.436 & 1.332 & 5.419 \\
\hline & & & Proposed & 3.811 & 8.575 & 1.361 & 5.935 & 3.622 & 7.440 & 1.332 & 5.421 \\
\hline & 1 & FOBT & Vo et al. [3] & 3.716 & 8.349 & 1.328 & 5.784 & 3.494 & 7.106 & 1.295 & 5.213 \\
\hline & & & Proposed & 3.716 & 8.353 & 1.327 & 5.785 & 3.495 & 7.111 & 1.295 & 5.216 \\
\hline & 10 & FOBT & Vo et al. [3] & 3.757 & 8.419 & 1.343 & 5.841 & 3.490 & 6.917 & 1.303 & 5.143 \\
\hline & & & Proposed & 3.757 & 8.423 & 1.343 & 5.843 & 3.491 & 6.922 & 1.303 & 5.145 \\
\hline
\end{tabular}

Tables 3, 4, and 5 show the robustness of the proposed element in the free vibration analysis for various types of cross-section and different boundary conditions. To investigate the asymmetric effect of cross-section on free vibration analysis, the beam is studied with simple-simple and pinned-sliding support conditions. The values of the free vibration frequencies of the beam with a symmetrical cross-section in the simple-simple and pinned-sliding support conditions are the same. Fig. 12 shows the ratios of the free vibration frequencies of the beam with a cross-section of $C$ (1-2-1) in the simple-simple condition to their values in the pinned-sliding support conditions.

In this figure, the results are coming for various values of and different length-to-thickness ratios. The ratio of the natural frequency of the beam with the asymmetrical cross-section in the simple-simple support condition to the pinned-sliding one increases with the change of the $L / h$ parameter from 10 to 5 . In the length-to-thickness ratios of 10,100 , and 1000 for the beam, the ratios of natural frequencies $\left(\omega_{S-S}\right) /\left(\omega_{S-P}\right)$ don't have a significant difference. Based on Fig. 12, by closing the possibility of horizontal movement of the two ends of the beam, their natural frequency values increases. This increase is greater for larger values of $k$.

\section{Conclusions}

In this paper, a simple, efficient element was first formulated based on the Timoshenko beam theory. For this purpose, third-order polynomials for the vertical displacement field of the beam and second-order polynomials for the axial displacement and rotation fields of the beam were used in this formulation. Satisfying the equilibrium

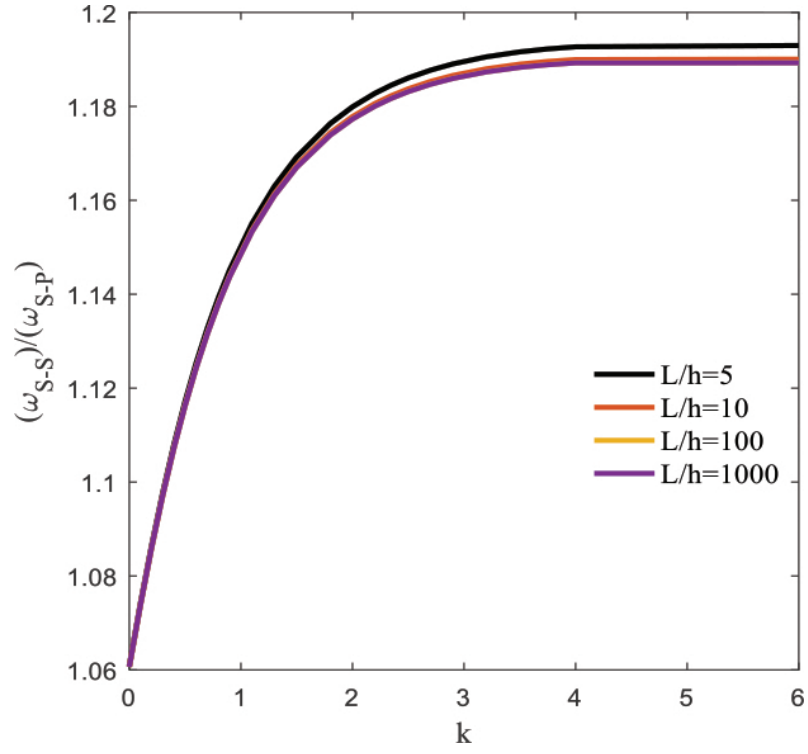

Fig. 12 The ratios of free-vibration frequencies of the beam with cross-section $C$ (1-2-1) in the simple-simple condition to their values in pinned-sliding one

equation, in addition to raising the efficiency of the element, reduced the number of unknowns. Based on the obtained shape functions, mass and stiffness matrices were derived for beams with changing material properties in the direction of thickness. Subsequently, the ability of the proposed element in free vibration and bending analysis of sandwich FG beams was assessed. In both free vibration and bending analysis, the rapid convergence rate of the proposed element was first proved. Comparing the good elements' responses of other researchers with the results of the proposed element under different boundary conditions and various aspect ratios revealed the high accuracy of the new element in bending and free vibration 
analysis of the beam with all cross-sections of $A, B$, and $C$. After validation of the new element, the asymmetric effect of the beam cross-section was studied. For this purpose, cross-section $C$ (1-2-1) was used. By increasing the parameter $k$, the asymmetry of the cross-section increases. For the beam under vertical loads, the asymmetry of the cross-section creates additional axial deformations in

\section{Reference}

[1] Apetre, N. A., Sankar, B. V., Ambur, D. R. "Analytical Modeling of Sandwich Beams with Functionally Graded Core", Journal of Sandwich Structures \& Materials, 10(1), pp. 53-74, 2008. https://doi.org/10.1177/1099636207081111

[2] Mohanty, S. C., Dash, R. R., Rout, T. "Static and dynamic stability analysis of a functionally graded Timoshenko beam", International Journal of Structural Stability and Dynamics, 12(04), Article No. $1250025,2012$.

https://doi.org/10.1142/S0219455412500253

[3] Vo, T. P., Thai, H.-T., Nguyen, T.-K., Maheri, A., Lee, J. "Finite element model for vibration and buckling of functionally graded sandwich beams based on a refined shear deformation theory", Engineering Structures, 64, pp. 12-22, 2014.

https://doi.org/10.1016/j.engstruct.2014.01.029

[4] Trinh, L. C., Vo, T. P., Osofero, A. I., Lee, J. "Fundamental frequency analysis of functionally graded sandwich beams based on the state space approach", Composite Structures, 156, pp. 263-275, 2016.

https://doi.org/10.1016/j.compstruct.2015.11.010

[5] Nguyen, T.-K., Nguyen, T. T.-P., Vo, T. P., Thai, H.-T. "Vibration and buckling analysis of functionally graded sandwich beams by a new higher-order shear deformation theory", Composites Part B: Engineering, 76, pp. 273-285, 2015.

https://doi.org/10.1016/j.compositesb.2015.02.032

[6] Sayyad, A. S., Ghugal, Y. M. "Modeling and analysis of functionally graded sandwich beams: a review", Mechanics of Advanced Materials and Structures, 26(21), pp. 1776-1795, 2019. https://doi.org/10.1080/15376494.2018.1447178 comparison to the symmetrical cross-section. So, rising the parameter of $k$ causes more additional axial deformations. In the bending analysis of beams with asymmetric cross-section, thin beams have more additional vertical displacement than thick beams. But, in free vibration analysis of beams, the effect of asymmetry on natural frequency of thick beams is higher than thin beams.

[7] Koutoati, K., Mohri, F., Daya, E. M. "Finite element approach of axial bending coupling on static and vibration behaviors of functionally graded material sandwich beams", Mechanics of Advanced Materials and Structures, 2019.

https://doi.org/10.1080/15376494.2019.1685144

[8] Li, W., Ma, H., Gao, W. "A higher-order shear deformable mixed beam element model for accurate analysis of functionally graded sandwich beams", Composite Structures, 221, Article No. 110830, 2019.

https://doi.org/10.1016/j.compstruct.2019.04.002

[9] Lengyel, G., Németh, R. K. "Symmetric free vibration of a cracked, quasi-continuous, masonry arch", Meccanica, 53, pp. 1071-1091, 2018.

https://doi.org/10.1007/s11012-017-0776-0

[10] Rezaiee-Pajand, M., Gharaei-Moghaddam, N. "Vibration and static analysis of cracked and non-cracked non-prismatic frames by force formulation", Engineering Structures, 185, pp. 106-121, 2019. https://doi.org/10.1016/j.engstruct.2019.01.117

[11] Ton-That, H. L., Nguyen-Van, H. "A Combined Strain Element in Static, Frequency and Buckling Analyses of Laminated Composite Plates and Shells", Periodica Polytechnica Civil Engineering, 65(1), pp. 56-71, 2021. https://doi.org/10.3311/PPci.16809

[12] Şimşek, M. "Fundamental frequency analysis of functionally graded beams by using different higher-order beam theories", Nuclear Engineering and Design, 240(4), pp. 697-705, 2010.

https://doi.org/10.1016/j.nucengdes.2009.12.013

\section{Appendix A}

$$
\left\{\begin{array}{c}
\bar{N}_{w 1}=-\frac{(3 x-l)(3 x-2 l)(x-l)}{2 l^{3}}, \bar{N}_{w 2}=\frac{9 x(3 x-2 l)(x-l)}{2 l^{3}} \\
\bar{N}_{w 4}=-\frac{9 x(3 x-l)(x-l)}{2 l^{3}}, \bar{N}_{w 5}=\frac{x(3 x-l)(3 x-2 l)}{2 l^{3}} \\
\bar{N}_{\phi 1}=N_{u 1}=(2 x-l)(x-l) / l^{2}, \bar{N}_{\phi 3}=N_{u 2}=-4 x(x-l) / l^{2} \\
\bar{N}_{\phi 5}=N_{u 3}=x(2 x-l) / l^{2}
\end{array}\right.
$$

\section{Appendix B}

$$
\begin{gathered}
N_{\omega 1}=\frac{(x-1)\left(2 x^{2}-x l-\delta\right)}{l \delta}, N_{\omega 2}=\frac{x(x-1)\left(2 x l-l^{2}-\delta\right)}{2 l \delta} \\
N_{\omega 3}=\frac{-x\left(2 x^{2}-3 x l+l^{2}-\delta\right)}{l \delta}, N_{\omega 4}=\frac{x(x-1)\left(2 x l-l^{2}+\delta\right)}{2 l \delta} \\
N_{\phi 1}=\frac{6}{l \delta} x(x-l), N_{\phi 2}=\frac{(x-l)}{l \delta}(3 x l-\delta) \\
N_{\phi 3}=-\frac{6}{l \delta} x(x-l), N_{\phi 4}=\frac{x}{l \delta}\left(3 x l-3 l^{2}+\delta\right) \\
\lambda=\frac{E I}{f_{s} G A}, \quad \delta=l^{2}+12 \lambda
\end{gathered}
$$

